\title{
Association of IGF1 Single-Nucleotide Polymorphisms with Myopia in Chinese Children
}

\author{
Tianyu Cheng Equal first author, 1, 2 , Jingjing Wang ${ }^{\text {Equal first author, } 1 \text {, Shuyu Xiong }}{ }^{1,2}$, Bo Zhang $^{1}$, Qiangqiang Li ${ }^{3}$, Xun Xu $^{1,2}$, \\ Xiangui He ${ }^{\text {Corresp. } 1,2}$ \\ ${ }^{1}$ Department of Preventative Ophthalmology, Shanghai Eye Disease Prevention and Treatment Center,Shanghai Eye Hospital, Shanghai, China \\ 2 Department of Ophthalmology, Shanghai General Hospital, Shanghai Jiao Tong University, Shanghai Key Laboratory of Ocular Fundus Disease, Shanghai, \\ China \\ 3 Baoshan Center for Disease Prevention and Control, Shanghai, China \\ Corresponding Author: Xiangui He \\ Email address: xianhezi@163.com
}

Purpose: To investigate the association between insulin-like growth factor 1 (IGF1) single-nucleotide polymorphisms (SNPs) and myopia in a young Chinese population.

Methods: A total of 654 Chinese children aged 6-13 years from one primary school participated in our study and underwent a series of comprehensive ocular examinations, including cycloplegic refraction and measurements of axial length. Myopia was defined as a spherical equivalence (SE) $\leq-0.5 \mathrm{D}$ in the worse eye. In total, six tagging SNPs of IGF1 were genotyped using the PCR-LDR (Polymerase Chain ReactionLigation Detection Reaction) method. We tested four different genetic modes (the allele, dominant, recessive, and additive models) of these SNPs and used multivariate logistic regression to calculate the effect of SNPs on myopia. In addition, we conducted a haplotype analysis with a variable-sized slidewindow strategy.

Results: Overall, 281 myopic children and 373 non-myopic controls were included in the analysis. The SNP rs2162679 showed a statistical difference between the two groups in both the allele $(p=0.0474)$ and additive ( $p=0.0497)$ models. After adjusting for age and gender, children with the genotype AA in the SNP rs 2162679 had a higher risk of myopia than those with the genotype $G G(O R=2.219,95 \% \mathrm{Cl}=$ $1.218-4.039, p=0.009$ ). All haplotypes that varied significantly between the two groups contained the SNP rs2162679, and the four-SNP window rs5742653-rs2162679 had the lowest $p$ value (Chi square $=$ $5.768, p=0.0163)$. However, after permutation tests, none of the associations remained statistically significant.

Conclusion: The SNP rs2162679 in IGF1 was associated with myopia in a young Chinese population. The $\mathrm{G}$ allele in the SNP rs2162679 may protect against myopia. 


\section{Association of IGF1 Single-Nucleotide Polymorphisms with Myopia in Chinese Children}

Tianyu Cheng $\mathrm{PhD}^{1,2}$, Jingjng Wang $\mathrm{MM}^{2}$, Shuyu Xiong $\mathrm{PhD}^{1,2}$, Bo Zhang² B.Optom, Qiangqiang $\mathrm{Ii}^{3}$ B.Optom, Xun $\mathrm{Xu}^{1,2} \mathrm{MD}$, Xiangui $\mathrm{He}^{1,2} \mathrm{PhD}$

1. Department of Preventative Ophthalmology, Shanghai Eye Disease Prevention and Treatment Center, Shanghai Eye Hospital, Shanghai, 200040, China

2. Department of Ophthalmology, Shanghai General Hospital, Shanghai Jiao Tong University, Shanghai Key Laboratory of Ocular Fundus Disease, Shanghai, 200080, China

3. Baoshan Center for Disease Prevention and Control, Shanghai 201901, China

\section{Please address correspondence to the following:}

Xiangui He

Email: xianhezi@163.com

Address: Building 5, No. 1440 Hongqiao Road, Shanghai, 200032, China

Telephone: +8615000755422

\section{ABSTRACT}

Purpose: To investigate the association between insulin-like growth factor 1 (IGF1) singlenucleotide polymorphisms (SNPs) and myopia in a young Chinese population.

Methods: A total of 654 Chinese children aged 6-13 years from one primary school participated in our study and underwent a series of comprehensive ocular examinations, including cycloplegic refraction and measurements of axial length. Myopia was defined as a spherical equivalence (SE) $\leq-0.5 \mathrm{D}$ in the worse eye. In total, six tagging SNPs of $I G F I$ were genotyped using the PCR-LDR (Polymerase Chain Reaction-Ligation Detection Reaction) method. We tested four different genetic modes (the allele, dominant, recessive, and additive models) of these SNPs and used multivariate logistic regression to calculate the effect of SNPs on myopia. In addition, we conducted a haplotype analysis with a variable-sized slide-window strategy. Results: Overall, 281 myopic children and 373 non-myopic controls were included in the analysis. The SNP rs2162679 showed a statistical difference between the two groups in both the allele $(p=0.0474)$ and additive $(p=0.0497)$ models. After adjusting for age and gender, children with the genotype AA in the SNP rs2162679 had a higher risk of myopia than those with the genotype $\mathrm{GG}(\mathrm{OR}=2.219,95 \% \mathrm{CI}=1.218-4.039, p=0.009)$. All haplotypes that varied significantly between the two groups contained the SNP rs2162679, and the four-SNP window rs5742653-rs2162679 had the lowest $p$ value (Chi square $=5.768, p=0.0163$ ). However, after permutation tests, none of the associations remained statistically significant.

Conclusion: The SNP rs2162679 in $I G F 1$ was associated with myopia in a young Chinese population. The G allele in the SNP rs2162679 may protect against myopia.

Key words: Single-nucleotide polymorphisms (SNPs); Insulin-like growth factor 1 (IGF-1); Myopia; Association study; Children and adolescents. 


\section{INTRODUCTION}

43 Myopia is the most common refractive disorder worldwide, especially in Asian countries, such 44 as China, Japan, and Singapore, and its prevalence is rising(Ang et al. 2019; Morgan et al. 2012). 45 Holden predicted that by the year 2050, almost half of the global population would be myopic, 46 with $10 \%$ progressing to high myopia(Holden et al. 2016). High myopia could be closely associated with complications like glaucoma, cataracts, retinal detachment, macular hemorrhage, and choroidal neovascularization(Ikuno 2017), which is one of the leading causes of blindness and results in financial burden(Iwase et al. 2006; Xu et al. 2006).

Myopia is a complex disease affected by both genetic and environmental factors, whose etiology and pathogenesis remain unclear(Morgan et al. 2018). Previous studies have identified 26 myopia-related loci (MYP1-MYP26) through various methods, such as family-based linkage analyses, genome-wide association studies, and whole exon sequencing studies(Cui et al. 2017; Guo et al. 2015; Wang et al. 2017; Xiao et al. 2016). Some candidate genes are associated with high myopia, while some are related to moderate myopia(Zhang 2015). However, the genetic effect on myopia is an interactive process involving multi-genes and is heterogeneous among different populations.

59

In the MYP3 locus on the chromosome 12q23.2, the insulin-like growth factor $1(I G F 1)$ gene codes for IGF-1, which is similar to insulin in structure and takes part in many physiological process including aging, apoptosis, development, cellular growth, metabolism, protein translation, and differentiation(D'Mello et al. 1993; Werner \& Le Roith 2000). IGF-1 is a kind of single-chain peptide composed of 70 amino acids which was also known as somatomedin $\mathrm{C}$ (Savage 2013). It plays an important role in human growth and development through GH-IGF1 axis (growth hormone) (Furlanetto \& Cara 1986). IGF-1 is found to be expressed in most of the tissues and the locally produced IGF-1 may be more important than its circulating endocrine form produced by liver(Han et al. 1988; Le Roith et al. 2001; Yakar et al. 1999). During puberty and pre-puberty, which are critical times for refraction development, IGF-1 may also be the inner motivation for ocular growth. For instance, after an intravitreal injection of IGF-1, the axial length of chicks grew longer(Zhu \& Wallman 2009). In addition, the axial length of patients with Laron syndrome, a disease that causes congenital growth hormone resistance, became normal, to a degree, after IGF-1 supplementation(Bourla et al. 2006). These studies have suggested that IGF-1 could contribute to ocular growth and the progression of myopia.

Genetic studies have been used to genotype the single-nucleotide polymorphisms of $I G F 1$ and have shown that it is significantly associated with high or extreme myopia in Caucasian and 78 Chinese populations(Mak et al. 2012; Zhuang et al. 2012). However, these associations were not replicated in a Polish family cohort and in two Japanese studies(Miyake et al. 2013; Rydzanicz et al. 2011; Yoshida et al. 2013). Nevertheless, all previous studies focused on the relationship 
81 between single-nucleotide polymorphisms (SNPs) in IGFI and high myopia, whilst the

82 association of SNP in $I G F I$ with mild or moderate myopia, which usually occurs during puberty

83 and pre-puberty, remains unknown. Here, we aimed to show that genetic variants of IGFI

84 contributed to the development of myopia in a young Chinese Han population.

85 METHODS

86 Participants

87 We used random cluster sampling to recruit a study population of children in grades 1-6 of a

88 primary school in Baoshan District, Shanghai, China. All participants underwent comprehensive

89 ophthalmologic tests, including an ophthalmologist exam and measurements of axial length and

90 spherical power. All participants were divided into two groups according to their spherical

91 equivalence (SE), which was measured using an automated computer refractometer after

92 inducing cycloplegia (model: KR-8900; Topcon, Tokyo, Japan).

93 We induced cycloplegia by administering one drop of topical $0.5 \%$ proparacaine (Alcaine;

94 Alcon, USA), followed by two drops of $1 \%$ cyclopentolate (Cyclogyl; Alcon, Fort Worth, TX,

95 USA) in each eye, with a 5-minute interval between each drop. Pupil size and pupillary light

96 reflex were examined at least 30 minutes after the last drop of cyclopentolate. Cycloplegia was

97 defined as the absence of a pupillary light reflex and a pupil size larger than $6 \mathrm{~mm}$. We measured

98 axial length using an IOL-master (version 5.02; Carl Zeiss Meditec, Oberkochen, Germany). To

99 avoid the invasiveness of blood testing, we collected saliva to extract DNA and target SNP

100 genotyping. We calculated SE using the following formula: $\mathrm{SE}=$ sphere $+0.5 *$ cylinder. We

101 diagnosed myopia as $\mathrm{SE} \leq-0.50 \mathrm{D}$ in the worse eye. Anyone who had amblyopia, glaucoma,

102 cataracts, or other genetic diseases associated with myopia were excluded from our study.

103 This study was approved by the Institutional Review Board of Shanghai General Hospital,

104 Shanghai Jiaotong University and was performed in accordance with the principles of the

105 Declaration of Helsinki. Informed consent was obtained from all participants and their guardians.

106 SNP selection and genotyping

107 To select tagSNPs from $I G F 1$ genes, we used Haploview software version 4.2

108 (https://www.broadinstitute.org/haploview/haploview) on the Han Chinese in Beijing (CHB)

109 dataset from the 1000 Genomes Project (http://www.internationalgenome.org). Inclusion criteria

110 were as follows: minor allele frequency (MAF) $>0.1$ and pairwise $\mathrm{r}^{2}>0.8$. In total, six SNPs

111 were used in the analysis, including rs6214, rs5742653, rs4764697, rs12423791, rs2162679, and

112 rs5742612. Among these tagSNPs, rs6214 and rs12423791 were associated with high myopia in

113 previous studies.

114 We extracted DNA with a salivary genomic DNA rapid extraction kit (KL3216T-50, Shanghai

115 Ze Ye Biological Technology Company, China) from oral epithelial cells under standard

116 conditions. We performed genotyping for the selected tagSNPs using the PCR-LDR method 
117 (Polymerase Chain Reaction-Ligation Detection Reaction) with technical support from the

118 Shanghai Biowing Applied Biotechnology Company. We selected 5\% of samples for duplicate

119 detection through simple random sampling.

120 PCR-LDR method combines PCR and LDR together and could detect variations in SNPs by Taq

121 ligase. The primer sequences of these six tagSNPs for PCR were shown in table 1 and the

122 corresponding probe sequences for LDR were shown in table 2. PCR was conducted for the

123 amplification of selected SNP sites. The PCR reaction system $(20 \mu 1)$ contained $2 \mu 11 \times$ buffer,

$1240.6 \mu \mathrm{l} \mathrm{Mg} 2+(3 \mathrm{mmol} / \mathrm{L}), 2 \mu \mathrm{l} \mathrm{dNTPs}(2 \mathrm{mmol} / \mathrm{L}$ each $), 0.2 \mu \mathrm{l}$ Taq polymerase $(1 \mathrm{U}), 4 \mu \mathrm{l} 1 \times \mathrm{Q}-$

125 solution, $0.4 \mu \mathrm{l}$ primer mix, $9.8 \mu \mathrm{ul} \mathrm{ddH} 2 \mathrm{O}$ and $1 \mathrm{ml}$ genomic DNA of samples. The PCR program

126 on MJ PTC-200 (MJ Research, USA) was as followed: denaturing at $95^{\circ} \mathrm{C}$ for 2 min; then

127 denaturing at $94{ }^{\circ} \mathrm{C}$ for $30 \mathrm{~s}$, annealing at $56^{\circ} \mathrm{C}$ for $90 \mathrm{~s}$, extension at $65^{\circ} \mathrm{C}$ for $30 \mathrm{~s}$, repeat 40

128 cycles; afterwards, extension for $10 \mathrm{~min}$ at $65^{\circ} \mathrm{C}$. Then multiplex LDR was performed for SNPs

129 detection. The LDR reaction system $(10 \mu \mathrm{l})$ consist of $1 \mu 11 \times$ buffer, $1 \mu l$ probe mix, $0.05 \mu 1$ Taq

130 DNA ligase (2 U), 3.95ul ddH2O and $4 \mu$ amplification products. The LDR program on Gene

131 Amp PCR system (model 9600, Norwalk, CT.06859 USA) was set as followed: initial

132 denaturing at $95^{\circ} \mathrm{C}$ for $2 \mathrm{~min}$, followed by 40 cycles of denaturing at $94{ }^{\circ} \mathrm{C}$ for $15 \mathrm{~s}$, and

133 annealing at $50^{\circ} \mathrm{C}$ for $25 \mathrm{~s}$. At last, Genemapper (ABI, Inc.) was applied for data analysis and

134 genotyping.

\section{Statistical analysis}

136 We analyzed clinical data using Statistical Product and Service Solutions (SPSS version 25,

137 IBM, USA) and genetic data using Haploview version 4.2 and gPLINK version 1.07. First, we

138 ensured that each SNP of the myopia and non-myopia groups passed the Hardy-Weinberg

139 equilibrium (HWE) test. We used a Chi Square test of four different genetic models (the allele,

140 dominant, recessive, and additive models) to show the distributions of different alleles and

141 genotypes as well as their potential genetic modes. While considering confounding factors, we

142 conducted a multivariate logistic regression analysis adjusting for age and gender. In addition,

143 we analyzed the associations between different haplotypes and myopia using a variable-sized

144 slide-window strategy in Haploview software. Significance was set at $p<0.05$, and we used the

14510000 times permutation test for multiple comparisons. The power of test was calculated using

146 two independent proportions power analysis (PASS version 11.0).

\section{RESULTS}

148 There were 281 myopes and 373 non-myopes with mean ages of $9.84 \pm 1.55$ years and $8.06 \pm$

1491.43 years, respectively. There were no significant differences in gender between the groups. The 150 mean SE of participants' right eye was $-2.55 \pm 1.64 \mathrm{D}$ in the myopia group and $0.84 \pm 0.81 \mathrm{D}$ in

151 the non-myopia group ( $p<0.001$ ). The mean axial length of participants' right eye was $24.58 \pm$

$1521.04 \mathrm{~mm}$ for the myopes and $23.02 \pm 0.79 \mathrm{~mm}$ for the non-myopes $(p<0.001)$. No significant

153 difference was observed in SE and axial length between right and left eyes (Table 3).

154 All six tagSNPs were successfully genotyped, and all of the duplicated samples showed the same 155 genotypes with their corresponding ones. The genotype distributions were all in line with HWE 
156 in both the myopia and non-myopia groups $(p>0.05)$. Their alleles and frequencies have been

157 shown in Table 4.

158 We compared differences in genotype between the two groups using four genetic models: the

159 allele, dominant, recessive, and additive models (Table 5). The SNP rs2162679 showed a

160 statistically significant association with myopia in the allele and additive models $(p=0.0474$ and

1610.04973 , respectively). However, these associations did not exist after we conducted the 10000

162 times permutation test. All the other SNPs were not significantly associated with myopia in these

163 four models. After adjusting for age and gender, participants with the genotype AA in the SNP

$164 \mathrm{rs} 2162679$ had a higher risk of myopia than those with the genotype $\mathrm{GG}(\mathrm{OR}=2.219,95 \% \mathrm{CI}=$

$1651.218-4.039, p=0.009$; Table 6 ), with post hoc power of $66.2 \%$. Also, the genotype CC in

166 rs12423791 and CT in rs5742612 were associated with a lower risk of myopia $(\mathrm{OR}=0.407$ and

1670.648 , respectively; 95\% CI $=0.185-0.896$ and $0.443-0.948$, respectively; $p=0.026$ ) after

168 adjusting for age and gender.

169 We performed a haplotype analysis between the myopia and non-myopia groups with a variable-

170 sized slide-window strategy in Haploview software version 4.2 (Table 7, Figure 1). The four-

171 SNP window rs5742653-rs2162679 had the lowest $p$ value (Chi square $=5.768, p=0.0163$ ), and

172 all SNP windows with $p$ values less than 0.05 included the SNP rs2162679. However, no

173 significant differences existed after the permutation test.

174 DISCUSSION

175 This study investigated the association between the SNP rs2162679 in the intron of IGF1 and

176 myopia in a young Chinese population during puberty and pre-puberty. Those with the genotype

177 AA in rs2162679 had a higher risk of myopia than those with the genotype GG (OR = 2.219).

178 The $\mathrm{A}>\mathrm{G}$ variation in this position may protect against myopia. In anther word, individuals

179 with G allele in rs2162679 of IGF1 have less chance of getting myopia. During puberty and pre-

180 puberty, increasing levels of hormones, such as growth factor and IGF-1, accelerate ocular

181 growth and the development of body stature. These genetic variants in IGF1 may be related to

182 the onset or early development of myopia in young populations.

183

184 We found no significant association between rs6214, rs12423791, and myopia in this young

185 Chinese population. However, within the MYP3 locus, these SNPs in IGF1 were previously

186 associated with high myopia, a finding that has been replicated by several studies. Metlapally et

187 al. were the first to test the SNPs in IGF1 and found that rs6214 was significantly related to any

188 type of myopia, including high myopia, in Caucasian populations(Metlapally et al. 2010).

189 However, this conclusion was not confirmed in a Polish family cohort or in two Chinese and two

190 Japanese studies(Miyake et al. 2013; Rydzanicz et al. 2011; Yoshida et al. 2013). A 2017 meta-

191 analysis including eight case-control studies published before June 2016 reported no significant

192 association between high myopia and the SNP rs6214 in IGF1(Zhang et al. 2017). Moreover,

193 Zhuang et al. found that rs12423791, another SNP in IGF1, was associated with extreme myopia

194 rather than high myopia in a Chinese population and that the haplotype containing this SNP

Peer) reviewing PDF | (2019:08:40662:1:0:CHECK 2 Dec 2019) 
195 showed a significant association with extreme myopia(Mak et al. 2012; Zhuang et al. 2012).

196 However, Yoshida et al., Miyake et al., and a 2017 Chinese study failed to replicate these

197 associations (Miyake et al. 2013; Wang et al. 2017; Yoshida et al. 2013). This may be due to

198 ethnic differences or the severity of myopia.

199

200

Unlike the positive relationship found in our study, two previous Japanese studies found no

201 significant associations between rs2162679 in IGF1 and high or extreme myopia(Miyake et al.

202 2013; Yoshida et al. 2013). Participants in our study were children in the puberty or pre-puberty

203 stages, and most suffered from mild or moderate myopia. These findings suggested that

204 rs2162679 was only associated with mild and moderate myopia rather than high or extreme myopia. To date, six MYPs (MYP6-10 and MYP14) have been discovered and mapped in populations with mild and moderate myopia(Cui et al. 2017). Baltimore et al. mapped a myopiarelated region in chromosome 1p36 in an Ashkenazi Jewish population with a mean SE of -3.46 D (Wojciechowski et al. 2006). In addition, chromosome 22q12 was associated with moderate myopia in 44 large American Ashkenazi Jewish families (MSE = -4.67 D)(Stambolian et al. 2004). Even though myopia is a complex disease affected by environmental factors, and high myopia is thought to be hereditary, it is necessary consider genetic factors in mild and moderate myopia.

213

Though intravitreal injections of insulin or IGF-1 in chicks results in extreme axial extension, changes in the axial length in mammals are relatively limited(Feldkaemper et al. 2009; Zhu \& Wallman 2009). This may be due to variation in their ocular structures and metabolism pathways(Mathis \& Schaeffel 2007). There is cartilage in the sclera of chick eyes, which could be stimulated by IGF-1 via cartilage anabolism(Martel-Pelletier et al. 1998; Rada et al. 1991). In addition, this structure could support axial elongation in chicks better than in mammals, whose eyes do not contain cartilage. This may explain why IGF-1 resulted in extreme increases in axial length in chicks but has only been related to mild to moderate myopia in humans.

222

223

Interestingly, rs2162679 of IGF1 had been reported to be associated with several kinds of cancer, in which the allele $\mathrm{G}$ could also decrease the risk of cancer onset(Xu et al. 2019). It reminded us that IGF-1 might play similar roles in myopia and cancer. More studies are needed to investigate the correlation between myopia and cancer.

227

228 However, this study had some limitations. First, the sample size was relatively small. Small sample sizes are more likely to result in false positives than larger sample sizes. They also have lower statistical power to detect positive results(Yoshida et al. 2013). Second, environmental factors, such as lack of outdoor activity or too much near work, are involved in the development

232 of myopia, so we these may have confounded our analysis(Saw et al. 2000). Further studies with

233 large sample sizes and in different populations are needed, especially those that stratify 
234 populations according to levels of myopia. Environmental factors and gene-environment

235 interactions should also be taken into consideration. Last but not least, considering that

236 rs 2162679 locates in the intron region of $I G F 1$, whether its variation could lead to protein

237 function change remained unknown.

238

239

In conclusion, we found that the SNP rs2162679 in $I G F 1$ was significantly associated with

240

myopia in this young Chinese population, which provided clues for in-depth mechanism

241

interpretation that IGF-1 may play a regulating role in the progression of myopia shift. Besides,

242

it indicated that rs2162679 in IGF1 could be one potential genetic loci for myopic risk

243

prediction.

244

245

ACKNOWLEDGEMENT

246

We would like to thank all the subjects participating in this research.

247

248

249

250

251

252

253

254

255

256

257

258

259

260

261

262

263

264

265

266

267

268

269

270

271

272

\section{References}

Ang M, Wong CW, Hoang QV, Cheung GCM, Lee SY, Chia A, Saw SM, Ohno-Matsui K, and Schmetterer L. 2019. Imaging in myopia: potential biomarkers, current challenges and future developments. The British journal of ophthalmology. 10.1136/bjophthalmol-2018312866

Bourla DH, Laron Z, Snir M, Lilos P, Weinberger D, and Axer-Siegel R. 2006. Insulinlike growth factor I affects ocular development: a study of untreated and treated patients with Laron syndrome. Ophthalmology 113:1197.e1191-1195. 10.1016/j.ophtha.2005.12.023

Cui XK, Zhu KK, Zhou Z, Wan SM, Dong Y, Wang XC, Li J, Zhang J, Mu HM, Qin L, and Hu YZ. 2017. A novel frameshift mutation in CX46 associated with hereditary dominant cataracts in a Chinese family. International journal of ophthalmology 10:684-690. 10.18240/ijo.2017.05.04

D'Mello SR, Galli C, Ciotti T, and Calissano P. 1993. Induction of apoptosis in cerebellar granule neurons by low potassium: inhibition of death by insulin-like growth factor I and cAMP. Proceedings of the National Academy of Sciences of the United States of America 90:10989-10993. 10.1073/pnas.90.23.10989

Feldkaemper MP, Neacsu I, and Schaeffel F. 2009. Insulin acts as a powerful stimulator of axial myopia in chicks. Investigative ophthalmology \& visual science 50:13-23. 10.1167/iovs.08-1702

Furlanetto RW, and Cara JF. 1986. Somatomedin-C/insulin-like growth factor-I as a modulator of growth during childhood and adolescence. Hormone research 24:177-184. $10.1159 / 000180557$

Guo H, Tong P, Liu Y, Xia L, Wang T, Tian Q, Li Y, Hu Y, Zheng Y, Jin X, Li Y, Xiong W, Tang

Peer) reviewing PDF | (2019:08:40662:1:0:CHECK 2 Dec 2019) 
273

274

275

276

277

278

279

280

281

282

283

284

285

286

287

288

289

290

291

292

293

294

295

296

297

298

299

300

301

302

303

304

305

306

307

308

309

310

311

B, Feng Y, Li J, Pan Q, Hu Z, and Xia K. 2015. Mutations of P4HA2 encoding prolyl 4hydroxylase 2 are associated with nonsyndromic high myopia. Genetics in medicine: official journal of the American College of Medical Genetics 17:300-306. 10.1038/gim.2015.28

Han VK, Lund PK, Lee DC, and D'Ercole AJ. 1988. Expression of somatomedin/insulin-like growth factor messenger ribonucleic acids in the human fetus: identification, characterization, and tissue distribution. The Journal of clinical endocrinology and metabolism 66:422-429. 10.1210/jcem-66-2-422

Holden BA, Fricke TR, Wilson DA, Jong M, Naidoo KS, Sankaridurg P, Wong TY, Naduvilath TJ, and Resnikoff S. 2016. Global Prevalence of Myopia and High Myopia and Temporal Trends from 2000 through 2050. Ophthalmology 123:1036-1042. 10.1016/j.ophtha.2016.01.006

Ikuno Y. 2017. OVERVIEW OF THE COMPLICATIONS OF HIGH MYOPIA. Retina (Philadelphia, Pa) 37:2347-2351. 10.1097/iae.0000000000001489

Iwase A, Araie M, Tomidokoro A, Yamamoto T, Shimizu H, and Kitazawa Y. 2006. Prevalence and causes of low vision and blindness in a Japanese adult population: the Tajimi Study. Ophthalmology 113:1354-1362. 10.1016/j.ophtha.2006.04.022

Le Roith D, Bondy C, Yakar S, Liu JL, and Butler A. 2001. The somatomedin hypothesis: 2001. Endocrine reviews 22:53-74. 10.1210/edrv.22.1.0419

Mak JY, Yap MK, Fung WY, Ng PW, and Yip SP. 2012. Association of IGF1 gene haplotypes with high myopia in Chinese adults. Archives of ophthalmology (Chicago, Ill : 1960) 130:209-216. 10.1001/archophthalmol.2011.365

Martel-Pelletier J, Di Battista JA, Lajeunesse D, and Pelletier JP. 1998. IGF/IGFBP axis in cartilage and bone in osteoarthritis pathogenesis. Inflammation research : official journal of the European Histamine Research Society [et al] 47:90-100. 10.1007/s000110050288

Mathis U, and Schaeffel F. 2007. Glucagon-related peptides in the mouse retina and the effects of deprivation of form vision. Graefe's archive for clinical and experimental ophthalmology = Albrecht von Graefes Archiv fur klinische und experimentelle Ophthalmologie 245:267275. 10.1007/s00417-006-0282-x

Metlapally R, Ki CS, Li YJ, Tran-Viet KN, Abbott D, Malecaze F, Calvas P, Mackey DA, Rosenberg T, Paget S, Guggenheim JA, and Young TL. 2010. Genetic association of insulin-like growth factor-1 polymorphisms with high-grade myopia in an international family cohort. Investigative ophthalmology \& visual science 51:4476-4479. 10.1167/iovs.09-4912

Miyake M, Yamashiro K, Nakanishi H, Nakata I, Akagi-Kurashige Y, Tsujikawa A, Moriyama M, Ohno-Matsui K, Mochizuki M, Yamada R, Matsuda F, and Yoshimura N. 2013. Insulin-like growth factor 1 is not associated with high myopia in a large Japanese cohort. Molecular vision 19:1074-1081.

Morgan IG, French AN, Ashby RS, Guo X, Ding X, He M, and Rose KA. 2018. The epidemics of

Peer) reviewing PDF | (2019:08:40662:1:0:CHECK 2 Dec 2019) 
312

313

314

315

316

317

318

319

320

321

322

323

324

325

326

327

328

329

330

331

332

333

334

335

336

337

338

339

340

341

342

343

344

345

346

347

348

349

350

myopia: Aetiology and prevention. Progress in retinal and eye research 62:134-149. 10.1016/j.preteyeres.2017.09.004

Morgan IG, Ohno-Matsui K, and Saw SM. 2012. Myopia. Lancet 379:1739-1748. 10.1016/S01406736(12)60272-4

Rada JA, Thoft RA, and Hassell JR. 1991. Increased aggrecan (cartilage proteoglycan) production in the sclera of myopic chicks. Developmental biology 147:303-312.

Rydzanicz M, Nowak DM, Karolak JA, Frajdenberg A, Podfigurna-Musielak M, Mrugacz M, and Gajecka M. 2011. IGF-1 gene polymorphisms in Polish families with high-grade myopia. Molecular vision 17:2428-2439.

Savage MO. 2013. Insulin-like growth factors, nutrition and growth. World review of nutrition and dietetics 106:52-59. 10.1159/000342577

Saw SM, Chua WH, Wu HM, Yap E, Chia KS, and Stone RA. 2000. Myopia: gene-environment interaction. Annals of the Academy of Medicine, Singapore 29:290-297.

Stambolian D, Ibay G, Reider L, Dana D, Moy C, Schlifka M, Holmes T, Ciner E, and BaileyWilson JE. 2004. Genomewide linkage scan for myopia susceptibility loci among Ashkenazi Jewish families shows evidence of linkage on chromosome 22q12. American journal of human genetics 75:448-459. 10.1086/423789

Wang P, Liu X, Ye Z, Gong B, Yang Y, Zhang D, Wu X, Zheng H, Li Y, Yang Z, and Shi Y. 2017. Association of IGF1 and IGF1R gene polymorphisms with high myopia in a Han Chinese population. Ophthalmic genetics 38:122-126. 10.3109/13816810.2016.1145699

Werner H, and Le Roith D. 2000. New concepts in regulation and function of the insulin-like growth factors: implications for understanding normal growth and neoplasia. Cellular and molecular life sciences : CMLS 57:932-942. 10.1007/p100000735

Wojciechowski R, Moy C, Ciner E, Ibay G, Reider L, Bailey-Wilson JE, and Stambolian D. 2006. Genomewide scan in Ashkenazi Jewish families demonstrates evidence of linkage of ocular refraction to a QTL on chromosome 1p36. Human genetics 119:389-399. 10.1007/s00439-006-0153-X

Xiao X, Li S, Jia X, Guo X, and Zhang Q. 2016. X-linked heterozygous mutations in ARR3 cause female-limited early onset high myopia. Molecular vision 22:1257-1266.

Xu GP, Chen WX, Zhao Q, Zhou H, Chen SZ, and Wu LF. 2019. Association between the insulinlike growth factor 1 gene rs2195239 and rs2162679 polymorphisms and cancer risk: a meta-analysis. BMC medical genetics 20:17. 10.1186/s12881-019-0749-3

Xu L, Wang Y, Li Y, Wang Y, Cui T, Li J, and Jonas JB. 2006. Causes of blindness and visual impairment in urban and rural areas in Beijing: the Beijing Eye Study. Ophthalmology 113:1134.e1131-1111. 10.1016/j.ophtha.2006.01.035

Yakar S, Liu JL, Stannard B, Butler A, Accili D, Sauer B, and LeRoith D. 1999. Normal growth and development in the absence of hepatic insulin-like growth factor I. Proceedings of the National Academy of Sciences of the United States of America 96:7324-7329. 10.1073/pnas.96.13.7324

Peer) reviewing PDF | (2019:08:40662:1:0:CHECK 2 Dec 2019) 
351 Yoshida M, Meguro A, Yoshino A, Nomura N, Okada E, and Mizuki N. 2013. Association study 352 of IGF1 polymorphisms with susceptibility to high myopia in a Japanese population. 353 Clinical ophthalmology (Auckland, NZ) 7:2057-2062. 10.2147/opth.S52726

354 Zhang D, Zeng G, Hu J, McCormick K, Shi Y, and Gong B. 2017. Association of IGF1 355 polymorphism rs6214 with high myopia: A systematic review and meta-analysis. $356 \quad$ Ophthalmic genetics 38:434-439.10.1080/13816810.2016.1253105

357 Zhang Q. 2015. Genetics of Refraction and Myopia. Progress in molecular biology and $358 \quad$ translational science 134:269-279. 10.1016/bs.pmbts.2015.05.007

359 Zhu X, and Wallman J. 2009. Opposite effects of glucagon and insulin on compensation for 360 spectacle lenses in chicks. Investigative ophthalmology \& visual science 50:24-36. $361 \quad 10.1167 /$ iovs.08-1708

362 Zhuang W, Yang P, Li Z, Sheng X, Zhao J, Li S, Yang X, Xiang W, Rong W, Liu Y, and Zhang 363 F. 2012. Association of insulin-like growth factor-1 polymorphisms with high myopia in the Chinese population. Molecular vision 18:634-644.

365 
Table $\mathbf{1}$ (on next page)

Primer Sequences and PCR Length 
1 Table 1: Primer Sequences and PCR Length

SNPs Upper Primer sequence $\left(5^{\prime}-3^{\prime}\right)$ Lower Primer sequence $\left(5^{\prime}-3^{\prime}\right)$ PCR length

\begin{tabular}{cccc}
\hline rs6214 & TTCCCTCTCAACAAAACTTT & GCTTTCCTCCTTGGGGGATT & 147 \\
rs5742653 & CAGCCTGAGCAGCATAGTGA & GCTCACTACAGCGTCAACCT & 144 \\
rs4764697 & TGGTCCCTGGATGTTGTTTAG & CCTCATCGCCCACCAAAACT & 151 \\
rs12423791 & TAGGCCCCATCTCTTTGCTG & TTGCTGCCTCCTGTTCACAT & 128 \\
rs2162679 & TTGAACAGGAAAACCCCACT & ACTGCATTTTTCTCAACAAG & 147 \\
rs5742612 & CTTTGCCTCATCGCAGGAGA & ATTGGAAGACAGCACTCGGG & 149
\end{tabular}


Table 2 (on next page)

Probe Sequences and LDR Length 


\section{Probe peerJ

P-

rs6214_modify GTTAAGTCTGCAGAAGACTGTTTTTTTTTTTTTTTTTTTTTTTTTTTTTTTTTTTTTT-

FAM

$\begin{array}{ll}\text { rs6214_A } & \text { T } \\ & \text { T }\end{array}$

rs6214_G $\quad$ TTTT

rs5742653_modify P-TGGGACTGCAGGCATGCATTTTTTTTTTTTTTTTTTTTTTTTTTTTTT-FAM

rs5742653_A TTTTTTTTTTTTTTTTTTTTTTTTTTCCCACCTCAGCCTTCTGAGTAGT 97

rs5742653_G TTTTTTTTTTTTTTTTTTTTTTTTTTTTCCCACCTCAGCCTTCTGAGTAGC 99

rs4764697_modify P-TGTGCAAGACTGCTTGAGGCTTTTTTTTTTTTTTTTTTTTTTTTTTTTTTTT-FAM

rs4764697_C TTTTTTTTTTTTTTTTTTTTTTTTTTTTTTCAGCCTCCTCCATGATCGTGCTG 105

rs4764697_T TTTTTTTTTTTTTTTTTTTTTTTTTTTTTTTTCAGCCTCCTCCATGATCGTGCTA 107

rs 12423791_modify P-TCAGTATCACTATTTCCTCTTTTTTTTTTTTTTTTTTTTTTTTT-FAM

rs12423791_C TTTTTTTTTTTTTTTTTTTTTTTAATGTATCTTCAGAATGCTCAG 89

rs12423791_GＴTTTTTTTTTTTTTTTTTTTTTTTTAATGTATCTTCAGAATGCTCAC

rs2162679_modify P-CTACATAGCCCAAAACACTGTTTTTTTTTTTTTTTTTTTT-FAM

rs2162679_A TTTTTTTTTTTTTTTTTTTCCGCATGGAAATCTTCCACCCT 81

rs2162679_G TTTTTTTTTTTTTTTTTTTTTCCGCATGGAAATCTTCCACCCC 83

rs5742612_modify P-GGTGTGATCTCATTTCCTAGTTTTTTTTTTTTTTTTTTTTTTTTTTTTTT-FAM

rs5742612_C $\quad$ TTTTTTTTTTTTTTTTTTTTTTTTTTCTTGTCCCAGTTGCCAAGTGAGG 101

rs5742612_T $\quad$ TTTTTTTTTTTTTTTTTTTTTTTTTTTTTTCTTGTCCCAGTTGCCAAGTGAGA 103

Table 2: Probe Sequences and LDR Length

2

3 
Table 3 (on next page)

Characteristics of Study Participants 
Table 3: Characteristics of Study Participants

\begin{tabular}{lcccc}
\hline & & Myopes $(\mathrm{n}=281)$ & Non-Myopes $(\mathrm{n}=373)$ & $p$ \\
\hline Age (years) & & $9.84 \pm 1.55$ & $8.06 \pm 1.43$ & $<0.001$ \\
Males (\%) & & $159(56.6 \%)$ & $193(51.3 \%)$ & 0.182 \\
\multirow{2}{*}{ AL (mm) } & OD $^{\mathrm{a}}$ & $24.58 \pm 1.04$ & $23.02 \pm 0.79$ & $<0.001$ \\
& OS $^{\mathrm{b}}$ & $24.40 \pm 1.81$ & $23.00 \pm 0.80$ & $<0.001$ \\
MSE $^{\mathrm{c}}$ (D) & OD & $-2.55 \pm 1.64$ & $0.84 \pm 0.81$ & $<0.001$ \\
& OS & $-2.25 \pm 1.84$ & $0.88 \pm 0.83$ & $<0.001$ \\
\hline
\end{tabular}

1 a. OD: right eye; b. OS: left eye; c. MSE: mean spherical equivalence 2 


\section{Table 4 (on next page)}

Genotypes and Hardy-Weinberg Equilibrium $p$ Values in Myopic and Non-Myopic Children 
Table 4: Genotypes and Hardy-Weinberg Equilibrium $p$ Values in Myopic and Non-Myopic Children

\begin{tabular}{|c|c|c|c|c|c|c|c|}
\hline \multirow{2}{*}{ SNP } & \multirow{2}{*}{ Chromosome } & \multirow{2}{*}{ Position } & \multirow{2}{*}{ Alleles } & \multicolumn{2}{|c|}{$\mathrm{MAF}^{\mathrm{a}}$} & \multicolumn{2}{|c|}{$\mathrm{HWE}^{\mathrm{b}} p$ value } \\
\hline & & & & Myopia & Control & Myopia & Control \\
\hline rs6214 & 12 & 102399791 & $\mathrm{G}>\mathrm{A}$ & 0.459 & 0.488 & 0.572 & 0.051 \\
\hline rs5742653 & 12 & 102442081 & $\mathrm{~A}>\mathrm{G}$ & 0.471 & 0.472 & 0.068 & 0.579 \\
\hline rs4764697 & 12 & 102459394 & $\mathrm{C}>\mathrm{T}$ & 0.180 & 0.164 & 0.546 & 1 \\
\hline rs12423791 & 12 & 102465050 & $\mathrm{G}>\mathrm{C}$ & 0.241 & 0.267 & 0.590 & 1 \\
\hline rs2162679 & 12 & 102477481 & $\mathrm{~A}>\mathrm{G}$ & 0.321 & 0.374 & 0.848 & 0.787 \\
\hline rs5742612 & 12 & 102481086 & $\mathrm{~T}>\mathrm{C}$ & 0.273 & 0.302 & 1 & 0.062 \\
\hline
\end{tabular}

1 a. MAF: minor allele frequency; b. HWE: Hardy-Weinberg Equilibrium 


\section{Table 5 (on next page)}

Association Tests of Six SNPs Between Myopes and Non-Myopes 
Table 5: Association Tests of Six SNPs Between Myopes and Non-Myopes

\begin{tabular}{|c|c|c|c|c|c|}
\hline \multirow{2}{*}{ SNP } & \multicolumn{4}{|c|}{ Model } & \multirow{2}{*}{$\begin{array}{l}\text { Correctec } \\
p \text { value }^{\mathrm{a}}\end{array}$} \\
\hline & Allele & Dominant & Recessive & Additive & \\
\hline rs6214 & 0.2981 & 0.174 & 0.7255 & 0.2782 & 0.7569 \\
\hline rs5742653 & 0.9858 & 0.5636 & 0.5094 & 0.9853 & 1.0000 \\
\hline rs4764697 & 0.4496 & 0.361 & 0.8901 & 0.4441 & 0.9100 \\
\hline rs 12423791 & 0.1464 & 0.266 & 0.1597 & 0.1436 & 0.4587 \\
\hline rs2162679 & $0.0474 *$ & 0.07926 & 0.159 & $0.04973 *$ & 0.1761 \\
\hline rs5742612 & 0.2539 & 0.1089 & 0.7883 & 0.2396 & 0.6886 \\
\hline
\end{tabular}

*Chi Square test $p<0.05$

a. multiple comparison corrected using the 10000 times permutation test 
Table 6(on next page)

Association Tests Adjusted by Age and Gender in the Additive Model 
Table 4: Association Tests Adjusted by Age and Gender in the Additive Model

\begin{tabular}{ccccc}
\hline SNP & Genotype & OR & $95 \%$ CI & $P$ \\
\hline \multirow{2}{*}{ rs6214 } & AA & 0.784 & $(0.461,1.336)$ & 0.371 \\
& AG & 0.841 & $(0.547,1.294)$ & 0.432 \\
& GG & reference & $/$ & $/$ \\
rs5742653 & AA & 1.307 & $(0.760,2.247)$ & 0.333 \\
& AG & 1.294 & $(0.802,2.088)$ & 0.290 \\
rs4764697 & GG & reference & $/$ & $/$ \\
& CC & 1.298 & $(0.389,4.331)$ & 0.671 \\
rs12423791 & CT & 1.456 & $(0.425,4.982)$ & 0.550 \\
& TT & reference & $/$ & $/$ \\
& CC & 0.407 & $(0.185,0.896)$ & $0.026^{*}$ \\
rs2162679 & CG & 0.775 & $(0.529,1.136)$ & 0.192 \\
& GG & reference & $/$ & $/$ \\
& AA & 2.219 & $(1.218,4.039)$ & $0.009^{*}$ \\
& AG & 1.529 & $(0.877,2.893)$ & 0.127 \\
rs5742612 & GG & reference & $/$ & $/$ \\
& CC & 0.523 & $(0.252,1.089)$ & 0.083 \\
& CT & 0.648 & $(0.443,0.948)$ & $0.026^{*}$ \\
\hline
\end{tabular}

*Logistic regression: $p<0.05$ after adjusting for age and gender 
Table 7 (on next page)

Haplotype Analysis Between Myopia and Non-Myopia Groups 
Table 7: Haplotype Analysis Between Myopia and Non-Myopia Groups

\begin{tabular}{|c|c|c|c|c|}
\hline Haplotypes & $\begin{array}{l}\text { Blocks with the } \\
\text { Lowest } p \text { Values }\end{array}$ & $\begin{array}{c}\text { Chi Square } \\
\text { Value }\end{array}$ & $p$ Value & $\begin{array}{c}\text { Corrected } p \\
\text { Value }^{\mathrm{a}}\end{array}$ \\
\hline \multicolumn{5}{|c|}{ Two-SNP window } \\
\hline rs6214-rs5742653 & $\mathrm{AA}$ & 1.14 & 0.2857 & 0.5587 \\
\hline rs5742653-rs4764697 & GT & 0.534 & 0.465 & 0.8216 \\
\hline rs4764697-rs 12423791 & $\mathrm{TC}$ & 2.003 & 0.157 & 0.3452 \\
\hline rs12423791-rs2162679 & GA & 5.394 & $0.0202 *$ & 0.0566 \\
\hline rs2162679-rs5742612 & $\mathrm{AT}$ & 3.134 & 0.0767 & 0.1970 \\
\hline \multicolumn{5}{|c|}{ Three-SNP window } \\
\hline rs6214-rs4764697 & $\mathrm{AAC}$ & 0.746 & 0.3878 & 0.8988 \\
\hline rs5742653-rs12423791 & GTC & 1.866 & 0.1719 & 0.5487 \\
\hline rs4764697-rs2162679 & CGG & 2.137 & 0.1438 & 0.4215 \\
\hline rs12423791-rs5742612 & GAT & 4.549 & $0.0329 *$ & 0.1536 \\
\hline \multicolumn{5}{|c|}{ Four-SNP window } \\
\hline rs6214-rs12423791 & AGCG & 1.456 & 0.2276 & 0.7044 \\
\hline rs5742653-rs2162679 & GCGA & 5.768 & $0.0163^{*}$ & 0.0586 \\
\hline rs4764697-rs5742612 & TGAT & 1.587 & 0.2077 & 0.7061 \\
\hline \multicolumn{5}{|c|}{ Five-SNP window } \\
\hline rs6214-rs2162679 & GGCGA & 3.99 & $0.0458^{*}$ & 0.1853 \\
\hline rs5742653-rs5742612 & GCGAT & 4.003 & $0.0454 *$ & 0.2245 \\
\hline \multicolumn{5}{|c|}{ Six-SNP window } \\
\hline rs6214-rs5742612 & GGCGAT & 2.937 & 0.0866 & 0.3999 \\
\hline
\end{tabular}

*Chi Square Test: $p<0.05$

a. multiple comparison corrected using the 10000 times permutation test 
Figure 1

Distribution of the tagging SNPs in IGF-1
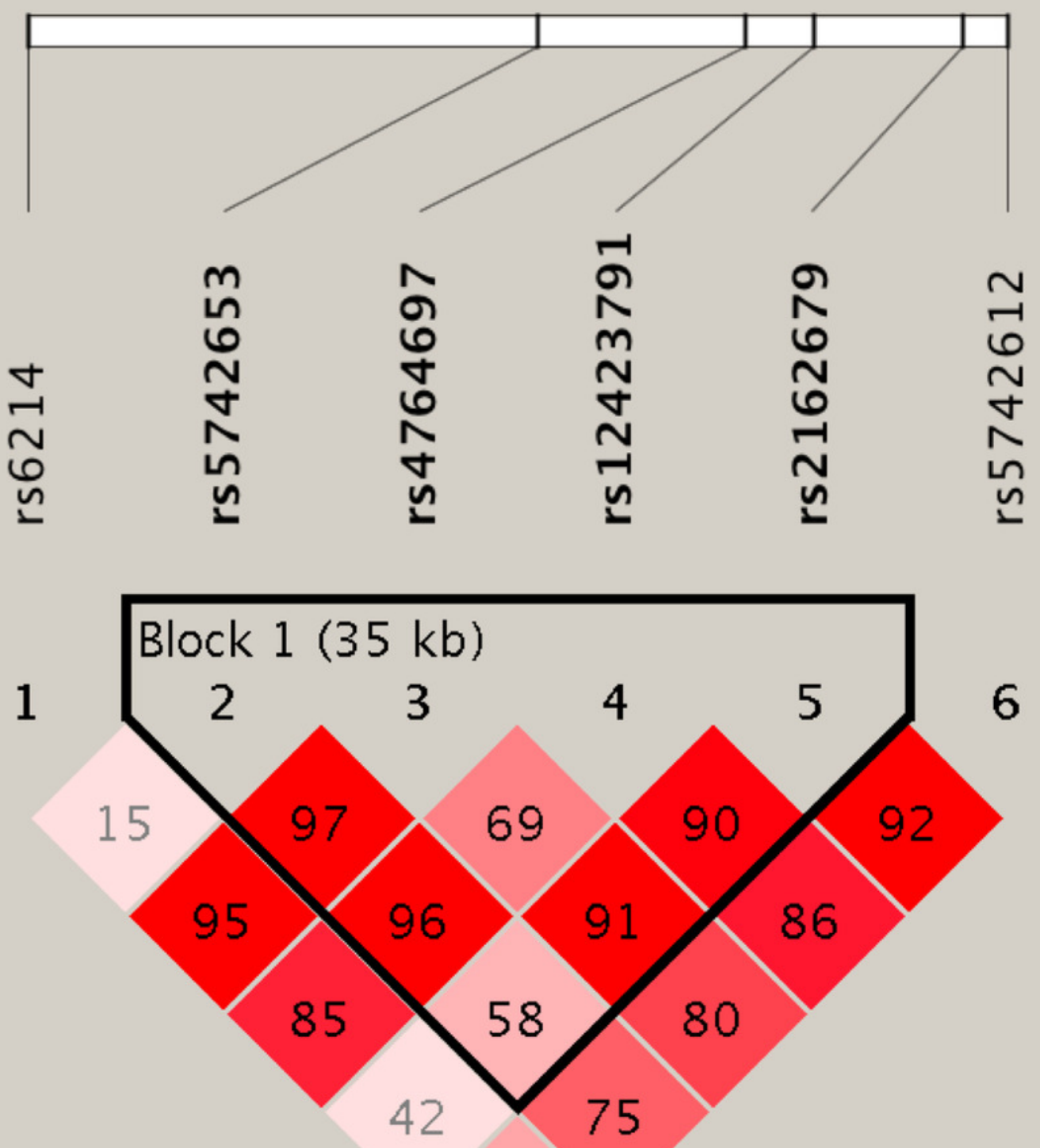

61 